\title{
The Burr XII Negative Binomial Distribution with Applications to Lifetime Data
}

\author{
Manoel Wallace A. Ramos ${ }^{1}$, Ana Percontini ${ }^{1}$, Gauss M. Cordeiro ${ }^{2} \&$ Ronaldo V. da Silva ${ }^{1}$ \\ ${ }^{1}$ Programa de Pós-Graduação em Matemática Computacional, Universidade Federal de Pernambuco, Brazil \\ ${ }^{2}$ Departamento de Estatística, Universidade Federal de Pernambuco, Brazil \\ Correspondence: Manoel Wallace A. Ramos, Programa de Pós-Graduação em Matemática Computacional, Uni- \\ versidade Federal de Pernambuco, Cidade Universitária, 50740-540 Recife, PE, Brazil. \\ E-mail: wallace.ifpb@gmail.com
}

Received: December 26, 2014 Accepted: January 12, 2015 Online Published: January 17, 2015

doi:10.5539/ijsp.v4n1p109 URL: http://dx.doi.org/10.5539/ijsp.v4n1p109

\begin{abstract}
A five-parameter model, called the Burr XII negative binomial distribution, is defined and studied. The new model contains as special cases some important lifetime distributions discussed in the literature, such as the log-logistic, Weibull, Pareto type II and Burr XII distributions, among several others. We derive the ordinary and incomplete moments, generating and quantile functions, mean deviations, reliability and two types of entropy. The order statistics and their moments are investigated. The method of maximum likelihood is proposed for estimating the model parameters. We obtain the observed information matrix. An application to real data demonstrates that the new distribution can provide a better fit than other classical lifetime models.
\end{abstract}

Keywords: Negative Binomial distribution, Burr XII distribution, Maximum likelihood, observed information matrix

\section{Introduction}

The negative binomial distribution has been widely used in mixing procedures of distributions. Several new models have been proposed and applied in survival analysis. Percontini et al. (2013) pionnered a family of univariate distributions generated by compouding the negative binomial distribution with any continuos model. For any baseline cumulative distribution function (cdf) $G(x)$, and $x \in \mathbb{R}$, they defined the G-Negative Binomial (G-NB) family of distributions with probability density function (pdf) $f(x)$ and cdf $F(x)$ given by

$$
f(x)=\frac{s \beta}{\left[(1-\beta)^{-s}-1\right]} g(x)\{1-\beta[1-G(x)]\}^{-s-1}
$$

and

$$
F(x)=\frac{(1-\beta)^{-s}-\{1-\beta[1-G(x)]\}^{-s}}{\left[(1-\beta)^{-s}-1\right]},
$$

respectively. The corresponding hazard rate function (hrf) is

$$
h(x)=\frac{s \beta g(x)\{1-\beta[1-G(x)]\}^{-s-1}}{\{1-\beta[1-G(x)]\}^{-s}-1} .
$$

The G-NB family has the same parameters of the G distribution plus two additional shape parameters $s>0$ and $\beta \in(0,1)$. If $\mathrm{X}$ is a random variable having pdf (1), we write $X \sim \mathrm{G}-\mathrm{NB}(s, \beta)$. This generalization is obtained by increasing the number of parameters compared to the $\mathrm{G}$ model, this increase being the price to pay for adding more flexibility to the generated distribution. A first positive point of the G-NB model is that it includes the G distribution as a sub-model when $s=1$ and $\beta \rightarrow 0$. A second one is that it includes as special cases important lifetime models published in recent years. 
Zimmer et al. (1998) introduced the three-parameter Burr XII distribution. This distribution, having logistic and Weibull as sub-models, is a very popular distribution for modeling lifetime data and phenomenon with monotone failure rates. When modeling monotone hazard rates, the Weibull distribution may be an initial choice because of its negatively and positively skewed density shapes. However, it does not provide a reasonable parametric fit for modeling phenomena with non-monotone failure rates such as the bathtub-shaped and the unimodal failure rates that are common in reliability and biological studies. Such bathtub hazard curves have nearly flat middle portions and the corresponding densities have a positive anti-mode. Unimodal failure rates can be observed in the course of a disease whose mortality reaches a peak after some finite period and then declines gradually.

In this paper, we use Percontini et al. (2013)'s generator to define a new model, so-called the Burr XII negative binomial (BXIINB) distribution, which generalizes the Burr XII (BXII) distribution.

The cdf and pdf of the BXII distribution are given by

$$
G(x ; c, k, a)=1-\left[1+\left(\frac{x}{a}\right)^{c}\right]^{-k} \text { and } g(x ; c, k, a)=c k a^{-c} x^{c-1}\left[1+\left(\frac{x}{a}\right)^{c}\right]^{-k-1},
$$

respectively, where $k>0$ and $c>0$ are shape parameters and $a>0$ is a scale parameter. The $n$th moment about zero of the BXII distribution (for $n<k c$ ) is given by

$$
\mu_{n}^{\prime}=a^{n} k B\left(k-n c^{-1}, n c^{-1}+1\right) \text {. }
$$

Some other mathematical properties of this distribution are discussed by Silva et al. (2008) and Paranaiba et al. (2011).

The BXII cdf has closed-form, thus it simplifies the computation of the percentiles and the likelihood function for censored data. This distribution has algebraic tails which are effective for modeling failures that occur with lesser frequency than with those models based on exponential tails. Hence, it represents a good distribution for modeling failure time data (Zimmer et al., 1998). Shao (2004a) discussed maximum likelihood estimation for the BXII distribution. Shao et al. (2004b) studied models for extremes using the BXII distribution with application to flood frequency analysis. According to Soliman (2005), this distribution covers the curve shape characteristics for a large number of distributions. The versatility and flexibility of the BXII distribution turns it quite attractive as a tentative model for fitting data real whose underlying distribution is unknown. Wu et al. (2007) studied the estimation problems for its model parameters based on progressive type II censoring with random removals, where the number of units removed at each failure time has a discrete uniform distribution. Silva et al. (2008) proposed a location-scale regression model based on this distribution, refereed to as the log-Burr XII regression model, which is a feasible alternative to the log-logistic regression model. Silva et al. (2011) defined a residual for the log-Burr XII regression model whose empirical distribution is close to normality. More recently, Paranaiba et al. (2011, 2012) studied the beta Burr XII and Kumaraswamy Burr XII distributions.

We define a new five-parameter BXIINB distribution inserting equations (3) into (1) and (2). Then, the pdf and cdf of the generated distribution (for $x>0$ ) are given by

$$
f(x)=\frac{s \beta c k a^{-c}}{\left[(1-\beta)^{-s}-1\right]} x^{c-1}\left[1+\left(\frac{x}{a}\right)^{c}\right]^{-k-1}\left\{1-\beta\left[1+\left(\frac{x}{a}\right)^{c}\right]^{-k}\right\}^{-s-1}
$$

and

$$
F(x)=\frac{(1-\beta)^{-s}-\left\{1-\beta\left[1+\left(\frac{x}{a}\right)^{c}\right]^{-k}\right\}^{-s}}{\left[(1-\beta)^{-s}-1\right]},
$$

respectively, where $a>0$ is a scale parameter and $s>0, c>0, k>0$ and $\beta \in(0,1)$ are shape parameters. Henceforth, the random variable $X$ having density function (5) is denoted by $X \sim \operatorname{BXIINB}(s, \beta, c, k, a)$.

Some possible shapes of the density function (5) for selected parameter values, including well-known distributions, are displayed in Figure 1.

The BXIINB hazard rate function (hrf) (for $x>0$ ) is given by

$$
h(x)=\frac{s \beta c k a^{-c} x^{c-1}\left[1+\left(\frac{x}{a}\right)^{c}\right]^{-k-1}\left\{1-\beta\left[1+\left(\frac{x}{a}\right)^{c}\right]^{-k}\right\}^{-s-1}}{-1+\left\{1-\beta\left[1+\left(\frac{x}{a}\right)^{c}\right]^{-k}\right\}^{-s}} .
$$


(a)

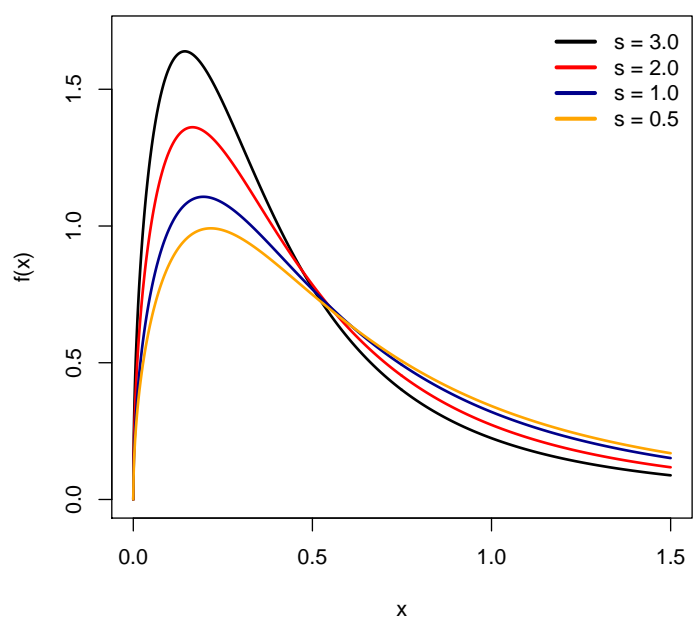

(c)

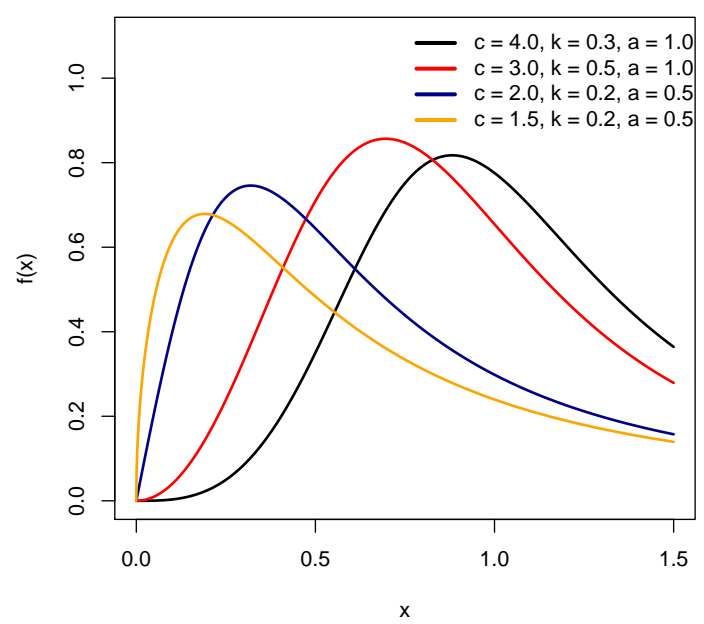

(b)

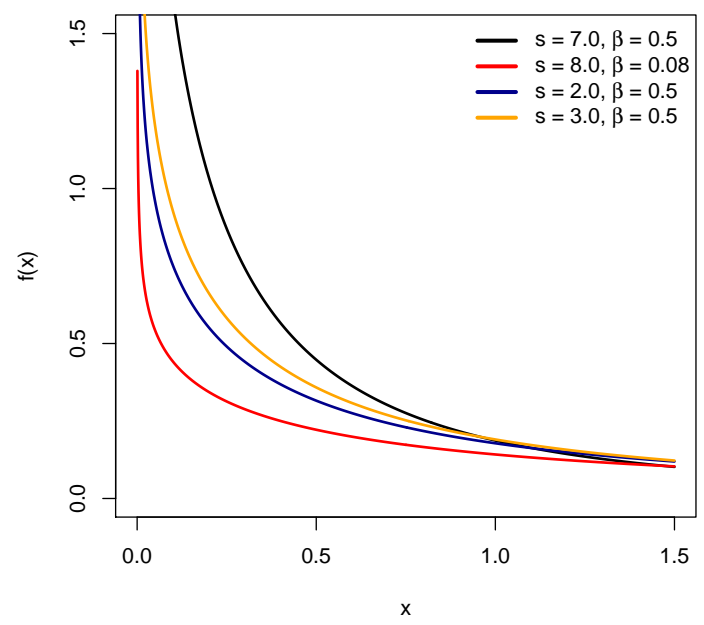

(d)

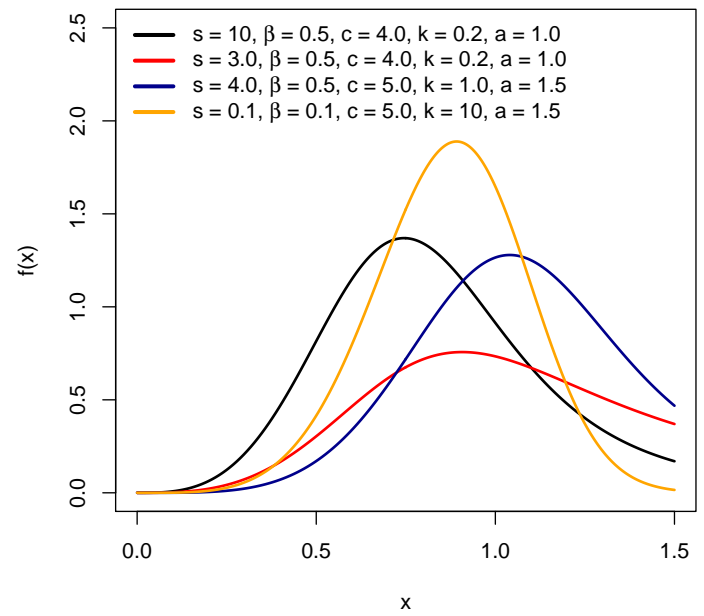

Figure 1. Plots of the BXIINB density function for some parameter values. (a) For $\beta=0.5, c=1.5, k=1.2$ and $a=1$. (b) For $c=0.8, k=0.5$ and $a=2$. (c) For $s=2$ and $\beta=0.5$.

Special BXIINB hazard rate shapes are displayed in Figure 2 for some parameter values. This hrf can be increasing, decreasing, constant and unimodal.

Some distributions arise as special cases of the BXIINB model:

- When $s=1$ and $\beta \rightarrow 0$, the BXII distribution is a limiting case of (5);

- For $s=1, \lambda \rightarrow 0, a=m^{-1}$ and $k=1$, it becomes the log-logistic (LL) distribution, whose survival function is $S(t ; m, c)=\left[1+(t m)^{c}\right]^{-1}$;

- For $s=1, \lambda \rightarrow 0$ and $c=1$, it reduces to the Pareto type II (PII) distribution;

- If $k \rightarrow \infty$ in addition to $s=1, \lambda \rightarrow 0$, it becomes the Weibull distribution;

- When $s=1$ in (6), we obtain the Burr XII geometric (BXIIG) distribution;

- For $s \rightarrow \infty$ and $\beta=\lambda / s$, equation (6) becomes the Burr XII Poisson (BXIIP) distribution. 
(a)

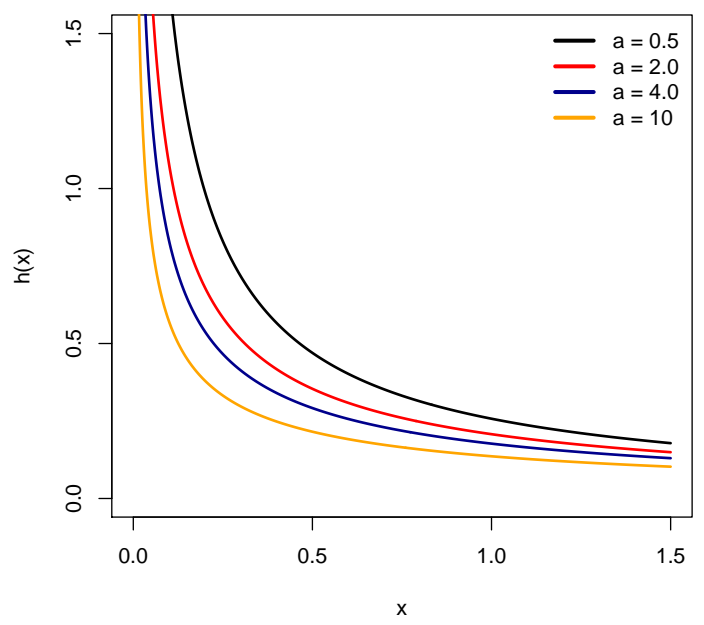

(c)

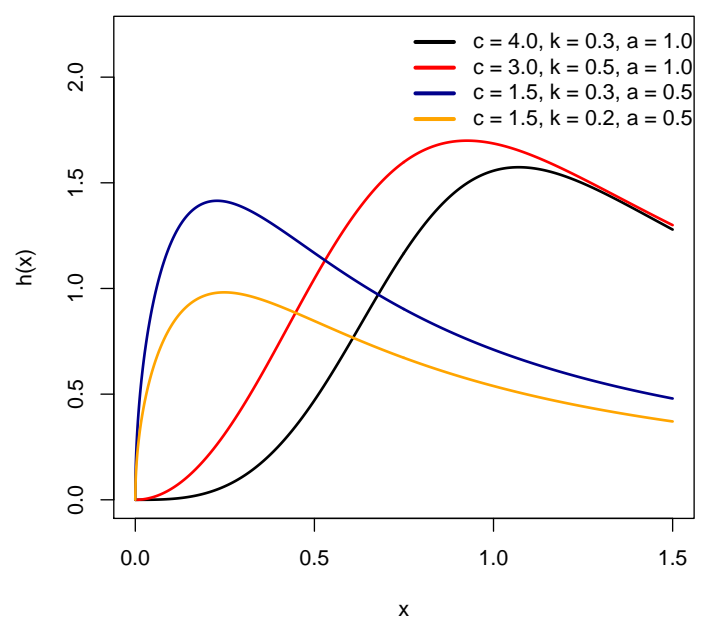

(b)

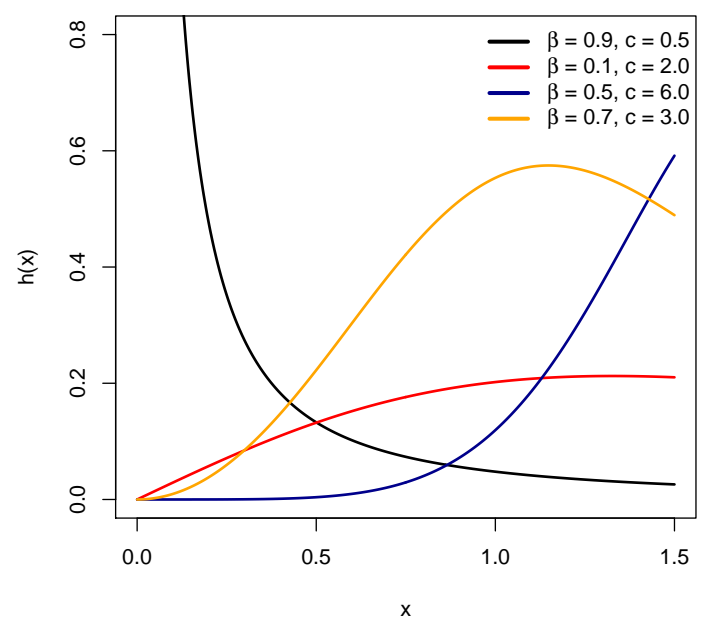

(d)

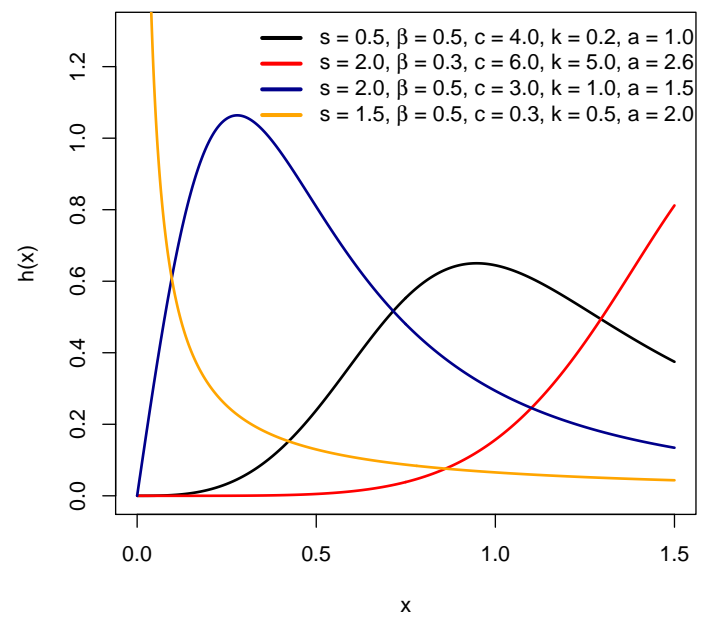

Figure 2. Plots of the BXIINB hrf for some parameter values. (a) For $s=2, \beta=0.5, c=0.5$ and $k=0.5$. (b) For $s=2, k=0.5$ and $a=2$. (c) For $s=3$ and $\beta=0.5$.

The BXIINB distribution is well-motivated for industrial applications and biological studies. As a first example, consider that the number, say $N$, of carcinogenic cells for an individual left active after an initial treatment follows a random variable $\mathrm{Z}$ with zero truncated negative binomial distribution and let $X_{i}$ be the time spent for the $i$ th carcinogenic cell to produce a detectable cancer mass, for $i \geq 1$. If $\left\{X_{1}, \ldots, X_{N}\right\}$ is a sequence of independent and identically distributed (iid) random variables independent of $N$ having the BXII distribution, then the time to relapse of cancer of a susceptible individual can be modeled by the BXIINB distribution. Another example considers that the failure of a device occurs due to the presence of an unknown number $N$ of initial defects of the same kind, which can be identifiable only after causing failure and are repaired perfectly. Define by $X_{i}$ the time to the failure of the device due to the $i$ th defect, for $i \geq 1$. If we assume that the $X_{i}$ 's are iid random variables independent of $N$, which follow a BXII distribution, then the time to the first failure is appropriately modeled by the BXIINB distribution. In fact, for reliability studies, the random variable $X=\operatorname{Min}\left\{X_{i}\right\}_{i=1}^{N}$ can be used in serial systems with identical components, which appear in many industrial applications and biological organisms.

In this paper, we study some mathematical properties of the BXIINB model and illustrate its potentiality. The 
paper is organized as follows. In Section 2, we demonstrate that the pdf of $\mathrm{X}$ can be expressed as a mixture of BXII densities. The quantile function is obtained in Section 3. Explicit expressions for the ordinary and incomplete moments, generating function and mean deviations are derived in Sections 4, 5 and 6, respectively. The Rényi and Shannon entropies and the reliability are determined in Sections 7 and 8, respectively. In Section 9 , we investigate the order statistics. Maximum likelihood estimation of the model parameters is performed and the observed information matrix is derived in Section 10. In Section 11, we illustrate the potentiality of the BXIINB distribution by means of a real data set. Finally, some conclusions are addressed in Section 12.

\section{Useful Expansion}

For any real $a$ and $|z|<1$, we have the binomial expansion

$$
(1-z)^{-a}=\sum_{k=0}^{\infty}(a)_{k} \frac{z^{k}}{k !}
$$

where $(a)_{0}=1$ and $(a)_{k}=a(a+1)(a+2) \ldots(a+k-1)=\Gamma(a+k) / \Gamma(a)$ is the ascending factorial. Using the power series (8), we can write (5) as

$$
f(x)=\sum_{i=0}^{\infty} \frac{c k s \beta^{i+1}(s+1)_{i}}{a^{c}\left[(1-\beta)^{-s}-1\right] i !} x^{c-1}\left[1+\left(\frac{x}{a}\right)^{c}\right]^{-k(1+i)-1},
$$

which gives an infinite linear combination

$$
f(x)=\sum_{i=0}^{\infty} \omega_{i}(s, \beta) g(x ; c,(i+1) k, a),
$$

where

$$
\omega_{i}(s, \beta)=\frac{s \beta^{i+1}(s+1)_{i}}{\left[(1-\beta)^{-s}-1\right](i+1) !}
$$

and $g(x ; c,(i+1) k, a)$ denotes the BXII density function with parameters $c,(i+1) k$ and $a$. We can verify that $\sum_{i=0}^{\infty} \omega_{i}(s, \beta)=1$. So, equation (9) reveals that the BXIINB density can be expressed as a linear combination of BXII densities. By integrating (9), we obtain

$$
F(x)=\sum_{i=0}^{\infty} \omega_{i}(s, \beta) G(x ; c,(i+1) k, a),
$$

where $G(x ; c,(i+1) k, a)$ denotes the BXII cumulative distribution with parameters $c,(i+1) k$ and $a$.

\section{Quantile Function}

Percontini et al. (2013) demonstrated that the G-NB quantile function can be expressed (for $0<u<1$ ) as

$$
Q(u)=Q_{G}\left\{1-\frac{1}{\beta}\left[1-(1-\beta)\left(1-u\left[1-(1-\beta)^{s}\right]\right)^{-\frac{1}{s}}\right]\right\},
$$

where $Q_{G}(u)$ is the baseline quantile function. The BXII quantile function is $Q_{B X I I}(u)=a\left[(1-u)^{-1 / k}-1\right]^{1 / c}$. Then, the BXIINB quantile function reduces to

$$
Q(u)=a\left\{\left[\frac{1}{\beta}\left\{1-\bar{\beta}\left[1-u\left(1-\bar{\beta}^{s}\right)\right]^{-\frac{1}{s}}\right\}\right]^{-1 / k}-1\right\}^{1 / c},
$$

where $\bar{\beta}=1-\beta$.

Quantiles of interest can be obtained from (12) by substituting appropriate values for $u$. In particular, the median of $X$ comes when $u=0.5$.

The motivation for using quantile-based measures is because of the non-existence of classical kurtosis for many generalized distributions. The Bowley's skewness is based on quartiles (Kenney and Keeping, 1962):

$$
B=\frac{Q(3 / 4)-2 Q(1 / 2)+Q(1 / 4)}{Q(3 / 4)-Q(1 / 4)}
$$


and the Moors kurtosis (Moors, 1988) is based on octiles:

$$
M=\frac{Q(7 / 8)-Q(5 / 8)-Q(3 / 8)+Q(1 / 8)}{Q(6 / 8)-Q(2 / 8)} .
$$

Plots of the skewness and kurtosis for the BXIINB distribution, for some choices of $\beta, c, k$ and $a$ as functions of $s$, and for some choices of $s, c, k$ and $a$ as functions of $\beta$ are displayed in Figure 3. These plots indicate that there is a great flexibility of the skewness and kurtosis curves for this distribution.
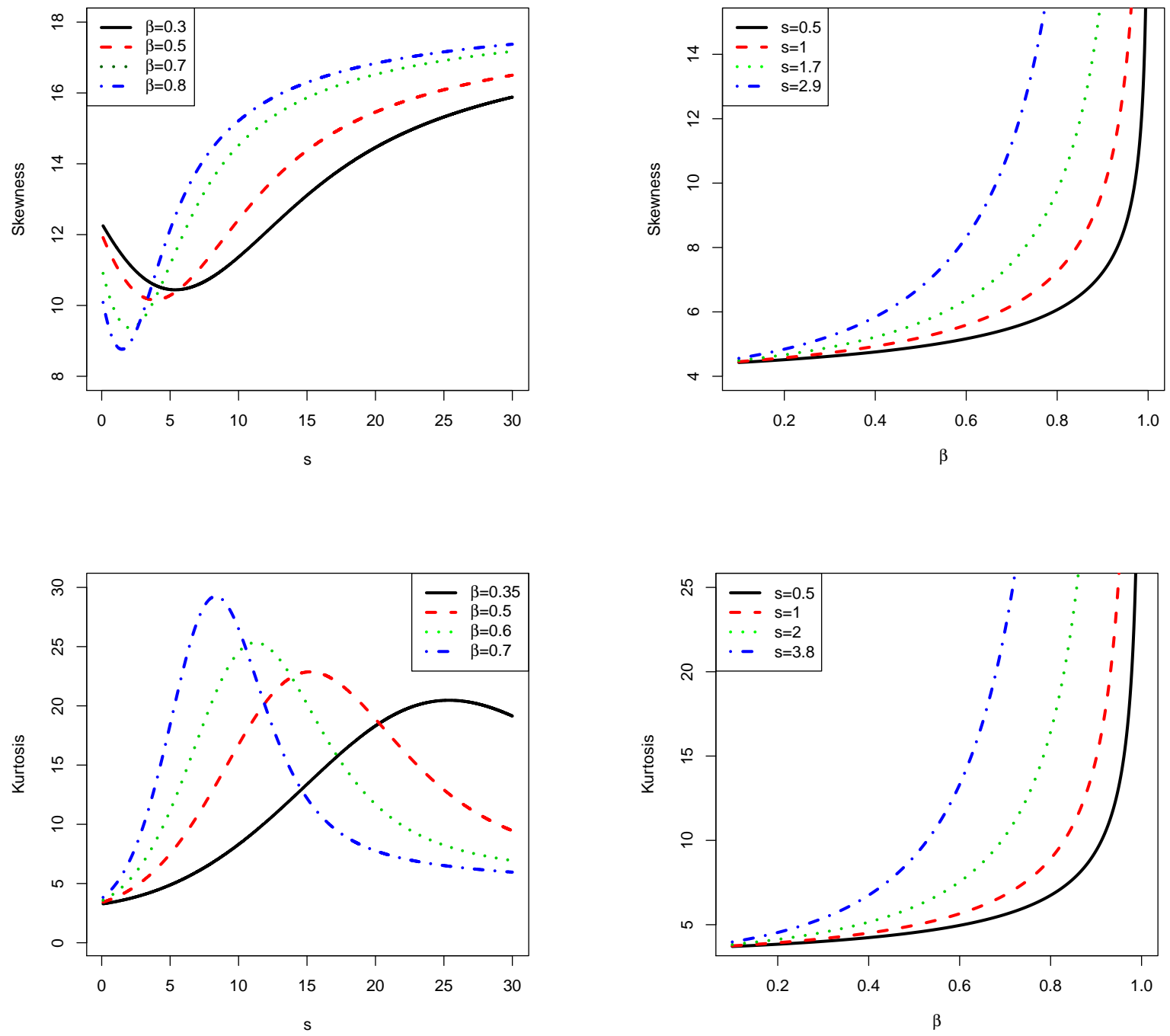

Figure 3. Plots of the BXIINB skewness and kurtosis as functions of $s$ for selected values of $\beta$ and as functions of $\beta$ for selected values of $s$.

\section{Moments}

The $n$th ordinary moment of $X$ can be obtained from equation (9) as

$$
E\left(X^{n}\right)=\sum_{i=0}^{\infty} \omega_{i}(s, \beta) E\left(Y_{i+1}^{n}\right)
$$


where $Y_{i+1}$ denotes the BXII random variable with density function $g(x ; c,(i+1) k, a)$. Thus, using (4) (for $\left.n<k c\right)$, we obtain

$$
E\left(X^{n}\right)=\sum_{i=0}^{\infty} \omega_{i}(s, \beta) a^{n}(i+1) k B\left[(i+1) k-\frac{n}{c}, 1+\frac{n}{c}\right] .
$$

The incomplete moments play an important role for measuring inequality, for example, income quantiles and Lorenz and Bonferroni curves. These curves depend on the first incomplete moment of the distribution. The $n$th incomplete moment of $X$ follows from equation (9) as

$$
\begin{aligned}
m_{n}(y) & =E\left(X^{n} \mid X<y\right)=\sum_{i=0}^{\infty} \omega_{i}(s, \beta) \int_{0}^{y} x^{n} g(x ; c,(i+1) k, a) d x \\
& =k c \sum_{i=0}^{\infty}(i+1) \omega_{i}(s, \beta) \int_{0}^{y} x^{n}\left[1+\left(\frac{x}{a}\right)^{c}\right]^{-k(i+1)-1} a^{-c} x^{c-1} d x .
\end{aligned}
$$

Setting $u=a^{-c} x^{c}$, the last equation becomes

$$
\begin{aligned}
m_{n}(y) & =k \sum_{i=0}^{\infty}(i+1) \omega_{i}(s, \beta) \int_{0}^{a^{-c} y^{c}} a^{n} u^{\frac{n}{c}}(1+u)^{-k(i+1)-1} d u \\
& =k \sum_{i=0}^{\infty} \frac{(i+1) c \omega_{i}(s, \beta)}{a^{c}(c+n)} y^{c+n}{ }_{2} F_{1}\left[1+(i+1) k, 1+\frac{n}{c}, 2+\frac{n}{c} ;-a^{-c} y^{c}\right],
\end{aligned}
$$

where ${ }_{2} F_{1}$ is the hypergeometric function defined by

$$
{ }_{2} F_{1}(a, b ; c ; x)=\sum_{k=0}^{\infty} \frac{(a)_{k}(b)_{k}}{(c)_{k}} \frac{x^{k}}{k !}
$$

and $(a)_{k}=a(a+1) \ldots(a+k-1)$ denotes the ascending factorial.

\section{Generating Function}

A first representation for the moment generating function (mgf) $M(t)$ of $X$ can be obtained from equation (9) as an infinite weighted sum

$$
M(t)=\sum_{i=0}^{\infty} \omega_{i}(s, \beta) M_{i+1}(t),
$$

where $M_{i+1}(t)$ is the $\operatorname{mgf}$ of the $\operatorname{BXII}(c,(i+1) k, a)$ distribution and $\omega_{i}(s, \beta)$ is defined by $(10)$. We provide a simple representation for the $\operatorname{mgf} M_{B X I I}(t)$ of the $\operatorname{BXII}(c, k, a)$ distribution. We can write for $t<0$

$$
M_{B X I I}(t)=c k \int_{0}^{\infty} \mathrm{e}^{a y t} y^{c-1}\left(1+y^{c}\right)^{-(k+1)} d y .
$$

Now, we require the Meijer $G$-function defined by

$$
G_{p, q}^{m, n}\left(x \mid \begin{array}{l}
a_{1}, \ldots, a_{p} \\
b_{1}, \ldots, b_{q}
\end{array}\right)=\frac{1}{2 \pi i} \int_{L} \frac{\prod_{j=1}^{m} \Gamma\left(b_{j}+t\right) \prod_{j=1}^{n} \Gamma\left(1-a_{j}-t\right)}{\prod_{j=n+1}^{p} \Gamma\left(a_{j}+t\right) \prod_{j=m+1}^{p} \Gamma\left(1-b_{j}-t\right)} x^{-t} d t
$$

where $\mathrm{i}=\sqrt{-1}$ is the complex unit and $L$ denotes an integration path (Gradshteyn and Ryzhik, 2000, Section 9.3). The Meijer $G$-function contains as particular cases many integrals with elementary and special functions (Prudnikov et al., 1986). 
We now assume that $c=m / k$, where $m$ and $k$ are positive integers. This condition is not restrictive since every positive real number can be approximated by a rational number. Using the integral (21) given in Appendix A, we conclude (for $t<0$ ) that

$$
M_{B X I I}(t)=m I\left(-a t, \frac{m}{k}-1, \frac{m}{k},-k-1\right) .
$$

Hence, from equation (15), the $\operatorname{mgf}$ of the $\operatorname{BXIINB}(s, \beta, c, k, a)$ distribution (for $t<0$ ) follows as

$$
M(t)=m \sum_{i=0}^{\infty} w_{i}(s, \beta) I\left(-a t, \frac{m}{(i+1) k}-1, \frac{m}{(i+1) k},-(i+1) k-1\right) .
$$

Equation (16) is the main result of this section.

\section{Mean Deviation}

The scatter in a population can be mesured by the totality of deviations from the mean and median. It is possible to derive the mean deviations about the mean and about the median from the relations $\delta_{1}(X)=E\left(\left|X-\mu_{1}^{\prime}\right|\right)=$ $2 \mu_{1}^{\prime} F\left(\mu_{1}^{\prime}\right)-2 m_{1}\left(\mu_{1}^{\prime}\right)$ and $\delta_{2}(X)=E(|X-M|)=\mu_{1}^{\prime}-2 m_{1}(M)$, respectively, where $\mu_{1}^{\prime}=E(X)$ comes from (13) with $n=1$, the median $M$ comes from (12) as

$$
Q(u)=a\left\{\left[\frac{1}{\beta}\left\{1-\bar{\beta}\left[1-1 / 2\left(1-\bar{\beta}^{s}\right)\right]^{-\frac{1}{s}}\right\}\right]^{-1 / k}-1\right\}^{1 / c},
$$

$F\left(\mu_{1}^{\prime}\right)$ is easily obtained from (6) and $m_{1}(\cdot)$ is determined from (14) with $n=1$. The first incomplete moment is useful to obtain the Bonferroni (B) and Lorenz (L) curves since they have applications in several areas. For a positive random variable $X$, they are defined by $B(\pi)=m_{1}(q) /\left(\pi \mu_{1}^{\prime}\right)$ and $L(\pi)=m_{1}(q) / \mu_{1}^{\prime}$, where $q=F^{-1}(\pi)=$ $Q(\pi)$ is calculated from (12). The measures $B(\pi)$ and $L(\pi)$ are determined from (14) by $m_{1}(Q(\pi))$.

\section{Entropies}

The entropy of a random variable $X$ with density function $f(x)$ is a measure of variation of the uncertainty. Two popular entropy measures are due to Shannon and Rényi (Shannon, 1951; Rényi, 1061). A large value of the entropy indicates the greater uncertainty in the random variable. The Rényi entropy is defined by (for $\delta>0$ and $\delta \neq 1)$

$$
I_{R}(\delta)=\frac{1}{(1-\delta)} \log \left(\int_{0}^{\infty} f^{\delta}(x) d x\right) .
$$

Based on the pdf (5), the Rényi entropy of $X$ is given by

$$
I_{R}(\delta)=\frac{J}{(1-\delta)}\left[\int_{0}^{\infty} x^{\delta(c-1)}\left[1+\left(\frac{x}{a}\right)^{c}\right]^{-\delta(k+1)}\left\{1-\beta\left[1+\left(\frac{x}{a}\right)^{c}\right]^{-k}\right\}^{-\delta(s+1)} d x\right],
$$

where $J=\left[\frac{s \beta c k a^{-c}}{(1-\beta)^{-s}-1}\right]^{\delta}$. Using (8), we obtain

$$
I_{R}(\delta)=\frac{J}{(1-\delta)}\left\{\sum_{i=0}^{\infty} \frac{[\delta(k+1)]_{i} \beta^{i}}{i !} \int_{0}^{\infty} x^{\delta(c-1)}\left[1+\left(\frac{x}{a}\right)^{c}\right]^{-\delta(k+1)-k i} d x\right\} .
$$

So, if $\delta>\left(k+1-c^{-1}\right)^{-1}$, the BXIINB Rényi entropy becomes

$$
I_{R}(\delta)=\frac{J}{(1-\delta)}\left\{a^{c+\delta} c^{-1} \sum_{i=0}^{\infty} \frac{[\delta(k+1)]_{i} \beta^{i}}{i !} B\left[\frac{\delta}{c}+1, \delta\left(k+1-c^{-1}\right)+k i-1\right]\right\} .
$$

The Shannon entropy of $X$ is given by

$$
\begin{aligned}
E\{-\log [f(X)]\}= & -\log \left(s \beta c k a^{-c}\right)+\log \left[(1-\beta)^{-s}-1\right] \\
& -(c-1) E[\log (X)]+(k+1) E\left\{\log \left[1+\left(\frac{X}{a}\right)^{c}\right]\right\} \\
& +(s+1) E\left(\log \left\{1-\beta\left[1+\left(\frac{X}{a}\right)^{c}\right]^{-k}\right\}\right) .
\end{aligned}
$$


Using (9), we have

$$
\begin{aligned}
E[\log (X)] & =\sum_{i=0}^{\infty} \omega_{i}(s, \beta) \int_{0}^{\infty} \log (x) g(x ; c,(i+1) k, a) d x \\
& =\sum_{i=0}^{\infty} \omega_{i}(s, \beta) k(i+1) \int_{0}^{\infty} c a^{-c} x^{c-1} \log (x)\left[1+\left(\frac{x}{a}\right)^{c}\right]^{-k(i+1)-1} d x .
\end{aligned}
$$

Setting $u=a^{c} x^{c}$, we obtain

$$
\begin{aligned}
E[\log (X)] & =\sum_{i=0}^{\infty} \omega_{i}(s, \beta) k(i+1) \int_{0}^{\infty} \log \left(a u^{\frac{1}{c}}\right)(1+u)^{-k(i+1)-1} d u \\
& =\frac{1}{c} \sum_{i=0}^{\infty} \omega_{i}(s, \beta)[\gamma+c \log (a)+\psi((i+1) k)],
\end{aligned}
$$

where $\gamma=0.5772 \ldots$ is the Euler's constant and $\psi(\cdot)$ is the digamma function.

Now, setting $v=1+a^{c} x^{c}$, we have

$$
\begin{aligned}
E\left\{\log \left[1+\left(\frac{X}{a}\right)^{c}\right]\right\} & =\sum_{i=0}^{\infty} \omega_{i}(s, \beta) k(i+1) \int_{1}^{\infty} \log (v) v^{-k(i+1)-1} d v \\
& =\sum_{i=0}^{\infty} \frac{\omega_{i}(s, \beta)}{k(i+1)}
\end{aligned}
$$

Analogously, setting $v=1+a^{c} x^{c}$, we obtain

$$
\begin{aligned}
E\left(\log \left\{1-\beta\left[1+\left(\frac{X}{a}\right)^{c}\right]^{-k}\right\}\right) & =\sum_{i=0}^{\infty} \omega_{i}(s, \beta) k(i+1) \int_{1}^{\infty} \log \left(1-\beta v^{-k}\right) v^{-k(i+1)-1} d v \\
& =\sum_{i=0}^{\infty} \omega_{i}(s, \beta) \beta \Phi(\beta, 1, i+2)+\log (1-\beta)
\end{aligned}
$$

where $\Phi(\cdot)$ is the Hurwitz-Lerch transcendent function given by $\Phi(z, s, \alpha)=\sum_{n=0}^{\infty} \frac{z^{n}}{(n+\alpha)^{s}}$. Then, we can express the Shannon entropy of $X$ as

$$
\begin{aligned}
E\{-\log [f(X)]\}= & -\log \left(s \beta c k a^{-c}\right)+\log \left[(1-\beta)^{-s}-1\right]-(c-1) \sum_{i=0}^{\infty} \omega_{i}(s, \beta) \\
& \times\left[\frac{\gamma+c \log (a)+\psi((i+1) k)}{c}\right]+(k+1) \sum_{i=0}^{\infty} \frac{\omega_{i}(s, \beta)}{k(i+1)} \\
& +(s+1) \sum_{i=0}^{\infty} \omega_{i}(s, \beta) \beta \Phi(\beta, 1, i+2)+\log (1-\beta) .
\end{aligned}
$$

\section{Reliability}

In the context of reliability, the stress-strength model describes the life of a component which has a random strength $X_{1}$ that is subjected to a random stress $X_{2}$. The component fails at the instant that the stress applied to it exceeds the strength, and the component will function satisfactorily whenever $X_{1}>X_{2}$. Hence, $R=\operatorname{Pr}\left(X_{2}<X_{1}\right)$ is a measure of component reliability. It has many applications especially in engineering concepts. Here, we obtain the reliability $R$ when $X_{1}$ and $X_{2}$ have independent $\operatorname{BXIINB}\left(s_{1}, \beta_{1}, c, k, a\right)$ and $\operatorname{BXIINB}\left(s_{2}, \beta_{2}, c, k, a\right)$ distributions. The reliability is defined by

$$
R=\int_{0}^{\infty} f_{1}(x) F_{2}(x) d x
$$

The pdf of $X_{1}$ and cdf of $X_{2}$ are obtained from equations (9) and (11) as

$$
f_{1}(x)=\sum_{m=0}^{\infty} \omega_{m}\left(s_{1}, \beta_{1}\right) g(x ; c,(m+1) k, a) \quad \text { and } \quad F_{2}(x)=\sum_{n=0}^{\infty} \omega_{n}\left(s_{2}, \beta_{2}\right) G(x ; c,(n+1) k, a),
$$


where

$$
\omega_{m}\left(s_{1}, \beta_{1}\right)=\frac{s_{1} \beta_{1}^{m+1}\left(s_{1}+1\right)_{m}}{\left[\left(1-\beta_{1}\right)^{-s_{1}}-1\right](m+1) !},
$$

and $\omega_{n}\left(s_{2}, \beta_{2}\right)$ comes analogously. Hence,

$$
\begin{aligned}
R= & \sum_{m, n=0}^{\infty} \omega_{m}\left(s_{1}, \beta_{1}\right) \omega_{n}\left(s_{2}, \beta_{2}\right) k(m+1) \int_{0}^{\infty} c a^{-c} x^{c-1}\left[1+\left(\frac{x}{a}\right)^{c}\right]^{-k(m+1)-1} \\
& \times\left\{1-\left[1+\left(\frac{x}{a}\right)^{c}\right]^{-k(n+1)}\right\} d x .
\end{aligned}
$$

Setting $u=a^{-c} x^{c}$, we have

$$
R=\sum_{m, n=0}^{\infty} \omega_{m}\left(s_{1}, \beta_{1}\right) \omega_{n}\left(s_{2}, \beta_{2}\right) k(m+1) \int_{0}^{\infty}(1+u)^{-k(m+1)-1}\left[1-(1+u)^{-k(n+1)}\right] d u .
$$

Hence, the BXIINB reliability is given by

$$
\begin{aligned}
R= & \sum_{m, n=0}^{\infty} \omega_{m}\left(s_{1}, \beta_{1}\right) \omega_{n}\left(s_{2}, \beta_{2}\right) k(m+1)\left\{-\frac{1}{k(m+n+2)}-\Gamma[k(n+1)+1]\right. \\
& \left.\times{ }_{2} \tilde{F}_{1}[1,(m+n+2) k+1 ;(n+1) k+2 ; 1]\right\},
\end{aligned}
$$

where $\Gamma(\cdot)$ is the gamma function and ${ }_{2} \tilde{F}_{1}(\cdot)$ is the regularized hypergeometric function defined by

$$
{ }_{2} \tilde{F}_{1}(a, b ; c ; x)=\frac{{ }_{2} F_{1}(a, b ; c ; x)}{\Gamma(c)} .
$$

\section{Order Statistics}

Order statistics make their appearance in many areas of statistical theory and practice. The density function $f_{i: n}(x)$ of the $i$ th order statistic, for $i=1, \ldots, n$, from iid random variables $X_{1}, \ldots, X_{n}$ having the G-NB distribution is given by (Percontini et al., 2013)

$$
f_{i: n}(x)=\sum_{r, v=0}^{\infty} \sum_{j=0}^{n-i} m_{r, v, j}(r+v+j+i) g(x) G(x)^{r+v+j+i},
$$

where

$$
\begin{gathered}
m_{r, v, j}=\frac{(-1)^{j} i(r+1) \omega_{r} \gamma_{j+i-1, v}}{(r+v+j+i)}\left(\begin{array}{c}
n \\
i
\end{array}\right)\left(\begin{array}{c}
n-i \\
j
\end{array}\right), \\
\omega_{r}=\frac{(-1)^{r} s}{(r+1)\left[(1-\beta)^{-s}-1\right]} \sum_{l=r}^{\infty} \frac{(s+1)_{l} \beta^{l+1}}{l !}\left(\begin{array}{l}
l \\
r
\end{array}\right), \\
\gamma_{j+i-1,0}=\omega_{0}^{j+i-1} \text { and } \gamma_{j+i-1, v}=\left(v \omega_{0}\right)^{-1} \sum_{m=1}^{v}[m(j+i)-v] \omega_{m} \gamma_{j+i-1, v-m} .
\end{gathered}
$$

So, applying (3) in equation (18), the density function $f_{i: n}(x)$ of the $i$ th order statistic, (for $\left.i=1, \ldots, n\right)$ from iid random variables $X_{1}, \ldots, X_{n}$ having the BXIINB distribution can be reduced to

$$
\begin{aligned}
f_{i: n}(x)= & \sum_{r, v=0}^{\infty} \sum_{j=0}^{n-i} m_{r, v, j}(r+v+j+i) \sum_{t=0}^{r+v+j+i-1}(-1)^{t} c k a^{-c} x^{c-1}\left(\begin{array}{c}
r+v+j+i-1 \\
t
\end{array}\right) \\
& \times\left[1+\left(\frac{x}{a}\right)^{c}\right]^{-k(t+1)-1} \\
= & \sum_{r, v=0}^{\infty} \sum_{j=0}^{n-i} \sum_{t=0}^{r+v+j+i-1} \phi_{t}(r, v, j) g(x ; c,(t+1) k, a),
\end{aligned}
$$


where

$$
\phi_{t}(r, v, j)=\frac{(-1)^{t}}{t+1}(r+v+j+i) m_{r, v, j}\left(\begin{array}{c}
r+v+j+i-1 \\
t
\end{array}\right) .
$$

Equation (19) reveals that the pdf of the BXIINB order statistics is a triple linear combination of BXII density functions. So, several mathematical quantities of these order statistics, such as the ordinary, incomplete and factorial moments, mgf and mean deviations, can be obtained from those BXII quantities. Clearly, the cdf of $X_{i: n}$ can be expressed as

$$
F_{i: n}(x)=\sum_{r, v=0}^{\infty} \sum_{j=0}^{n-i} \sum_{i=0}^{r+v+j+i-1} \phi_{t}(r, v, j) G(x ; c,(t+1) k, a) .
$$

For example, from equation (19), the moments of the BXIINB order statistics can be expressed directly in terms of the BXII moments (for $p<k c$ ) as

$$
\begin{aligned}
E\left(X_{i: n}^{p}\right) & =\sum_{r, v=0}^{\infty} \sum_{j=0}^{n-i} \sum_{t=0}^{r+v+j+i-1} \phi_{t}(r, v, j) \int_{0}^{\infty} x^{p} g(x ; c,(t+1) k, a) \\
& =\sum_{r, v=0}^{\infty} \sum_{j=0}^{n-i} \sum_{t=0}^{r+v+j+i-1} \phi_{t}(r, v, j)(t+1) k a^{p} B\left[(t+1) k-\frac{p}{c}, 1+\frac{p}{c}\right] .
\end{aligned}
$$

\section{Maximum Likelihood Estimation}

We determine the maximum likelihood estimates (MLEs) of the parameters of the BXIINB distribution from complete samples only. Let $x_{1}, \ldots, x_{n}$ be a random sample of size $n$ from the $\operatorname{BXIINB}(s, \beta, c, k, a)$ distribution. The $\log$-likelihood function for the vector of parameters $\boldsymbol{\theta}=(s, \beta, c, k, a)^{T}$ can be expressed as

$$
\begin{aligned}
l(\boldsymbol{\theta})= & n \log \left(s \beta c k a^{-c}\right)-n \log \left[(1-\beta)^{-s}-1\right]+(c-1) \sum_{i=1}^{n} \log (x) \\
& -(k+1) \sum_{i=1}^{n} \log \left[1+\left(\frac{x}{a}\right)^{c}\right]-(s+1) \sum_{i=1}^{n} \log \left\{1-\beta\left[1+\left(\frac{x}{a}\right)^{c}\right]^{-k}\right\} .
\end{aligned}
$$

The components of the score vector $U(\boldsymbol{\theta})$ are

$$
\begin{aligned}
& U_{s}(\boldsymbol{\theta})=\frac{n}{s}+\frac{n(1-\beta)^{-s} \log (1-\beta)}{(1-\beta)^{-s}-1}-\sum_{i=1}^{n} \log \left\{1-\beta\left[1+\left(\frac{x_{i}}{a}\right)^{c}\right]^{-k}\right\}, \\
& U_{\beta}(\boldsymbol{\theta})=\frac{n}{\beta}-\frac{n s(1-\beta)^{-s-1}}{(1-\beta)^{-s}-1}+(s+1) \sum_{i=1}^{n}\left\{\frac{\left[1+\left(\frac{x_{i}}{a}\right)^{c}\right]^{-k}}{1-\beta\left[\left(\frac{x_{i}}{a}\right)^{c}+1\right]^{-k}}\right\}, \\
& U_{c}(\boldsymbol{\theta})=\frac{n}{c}-n \log (a)+\sum_{i=1}^{n} \log \left(x_{i}\right)-(k+1) \sum_{i=1}^{n}\left[\frac{\left(\frac{x_{i}}{a}\right)^{c} \log \left(\frac{x_{i}}{a}\right)}{1+\left(\frac{x_{i}}{a}\right)^{c}}\right] \\
& -(s+1) \sum_{i=1}^{n}\left\{\frac{\beta k\left(\frac{x_{i}}{a}\right)^{c} \log \left(\frac{x_{i}}{a}\right)\left[1+\left(\frac{x_{i}}{a}\right)^{c}\right]^{-k-1}}{1-\beta\left[1+\left(\frac{x_{i}}{a}\right)^{c}\right]^{-k}}\right\} \text {, } \\
& U_{k}(\boldsymbol{\theta})=\frac{n}{k}-\sum_{i=1}^{n} \log \left[1+\left(\frac{x_{i}}{a}\right)^{c}\right]-(s+1) \sum_{i=1}^{n}\left\{\frac{\beta\left[1+\left(\frac{x_{i}}{a}\right)^{c}\right]^{-k} \log \left[1+\left(\frac{x_{i}}{a}\right)^{c}\right]}{1-\beta\left[1+\left(\frac{x_{i}}{a}\right)^{c}\right]^{-k}}\right\},
\end{aligned}
$$




$$
\begin{aligned}
U_{a}(\boldsymbol{\theta})= & -\frac{c n}{a}+(k+1) \sum_{i=1}^{n}\left\{\frac{c x_{i}\left(\frac{x_{i}}{a}\right)^{c-1}}{a^{2}\left[1+\left(\frac{x_{i}}{a}\right)^{c}\right]}\right\}+(s+1) \sum_{i=1}^{n}\left\{\frac{\beta c k x_{i}\left(\frac{x_{i}}{a}\right)^{c-1}}{a^{2}\left\{1-\beta\left[1+\left(\frac{x_{i}}{a}\right)^{c}\right]^{-k}\right\}}\right. \\
& \left.\times\left[1+\left(\frac{x_{i}}{a}\right)^{c}\right]^{-k-1}\right\} .
\end{aligned}
$$

Let $\widehat{\boldsymbol{\theta}}$ be the MLE of $\boldsymbol{\theta}$. The log-likelihood can be maximized either directly by using the Proc NLMixed of SAS or the sub-routine MaxBFGS of the Ox program (see, Doornik, 2007) or by solving the nonlinear likelihood equations $U_{s}(\boldsymbol{\theta})=U_{k}(\boldsymbol{\theta})=U_{c}(\boldsymbol{\theta})=U_{p}(\boldsymbol{\theta})=0$ simultaneously. For interval estimation and hypothesis tests on the model parameters, we require the $5 \times 5$ unit observed information matrix $J(\theta)=-\left\{U_{p q}\right\}$, where $p, q$ vary over the elements in $\boldsymbol{\theta}=(s, \beta, c, k, a)$. The elements $U_{p q}$ are given in Appendix B. Based on the approximate multivariate normal $N_{5}\left(0, J(\widehat{\boldsymbol{\theta}})^{-1}\right)$ distribution, we can construct confidence regions for the model parameters. We can compute the maximum values of the unrestricted and restricted log-likelihoods to obtain likelihood ratio (LR) statistics for testing some sub-models of the BXIINB distribution. Hypothesis tests of the type $H_{0}: \psi=\psi_{0}$ versus $H_{1}: \psi \neq \psi_{0}$, where $\psi$ is a vector formed with some components of $\boldsymbol{\theta}$ and $\psi_{0}$ is a specified vector, can be based on LR statistics. For example, the test of $H_{0}: s=1$ and $\beta \rightarrow 0$ versus $H_{1}: H_{0}$ is not true is equivalent to compare the BXIINB and BXII distributions and then the LR statistic reduces to

$$
w=2\{\ell(\widehat{s}, \widehat{\beta}, \widehat{c}, \widehat{k}, \widehat{a})-\ell(1,0, \widetilde{c}, \widetilde{k}, \widetilde{a})\},
$$

where $\widehat{s}, \widehat{\beta}, \widehat{c}, \widehat{k}$ and $\widehat{a}$ are the MLEs under $H_{1}$ and $\widetilde{c}, \widetilde{k}$ and $\widetilde{a}$ are the estimates under $H_{0}$.

\section{Application}

In this section, we illustrate the usefulness of the BXIINB distribution applied to a real data set. These data on failure times are reported in the book "Weibull Models" by Murthy et al. (2004, p. 297):

$0.040,1.866,2.385,3.443,0.301,1.876,2.481,3.467,0.309,1.899,2.610,3.478,0.557,1.911,2.625,3.578$, $0.943,1.912,2.632,3.595,1.070,1.914,2.646,3.699,1.124,1.981,2.661,3.779,1.248,2.010,2.688,3.924,1.281$, $2.038,2.82,3,4.035,1.281,2.085,2.890,4.121,1.303,2.089,2.902,4.167,1.432,2.097,2.934,4.240,1.480$, $2.135,2.962,4.255,1.505,2.154,2.964,4.278,1.506,2.190,3.000,4.305,1.568,2.194,3.103,4.376,1.615$, $2.223,3.114,4.449,1.619,2.224,3.117,4.485,1.652,2.229,3.166,4.570,1.652,2.300,3.344,4.602,1.757$, $2.324,3.376,4.663$.

We also fit the density functions of the Exponentiated Burr XII Poisson (Exp-BXIIP), Beta Burr XII (BBXII), Kumaraswamy Burr XII (KwBXII) and McDonald Burr XII (McBXII) distributions given by

$$
\begin{aligned}
& f_{\operatorname{Exp}-\mathrm{BXIIP}}(x ; c, k, a, \alpha, \lambda)=\frac{c k a^{-c} \alpha \lambda}{1-\mathrm{e}^{-\lambda}} x^{c-1}\left[1+\left(\frac{x}{a}\right)^{c}\right]^{-k-1}\left\{1-\left[1+\left(\frac{x}{a}\right)^{c}\right]^{-k}\right\}^{\alpha-1} \\
& \times \exp \left\{-\lambda\left[1-\left(1+\left(\frac{x}{a}\right)^{c}\right)^{-k}\right]^{\alpha}\right\} \\
& f_{\mathrm{BBXII}}(x ; c, k, a, d, b)=\frac{c k a^{-c}}{B(d, b)} x^{c-1}\left[1+\left(\frac{x}{a}\right)^{c}\right]^{-k b-1}\left\{1-\left[1+\left(\frac{x}{a}\right)^{c}\right]^{-k}\right\}^{d-1}, \\
& f_{\mathrm{KwBXII}}(x ; c, k, a, d, b)=d b c k a^{-c} x^{c-1}\left[1+\left(\frac{x}{a}\right)^{c}\right]^{-k-1}\left\{1-\left[1+\left(\frac{x}{a}\right)^{c}\right]^{-k}\right\}^{\alpha-1} \\
& \times\left\{1-\left\{1-\left[1+\left(\frac{x}{a}\right)^{c}\right]^{-k}\right\}^{d}\right\}^{b-1}
\end{aligned}
$$

and

$$
\begin{aligned}
f_{\mathrm{McBXIIP}}(x ; c, k, a, d, b, \alpha) & =\frac{c k a^{-c} \alpha}{B(d, b)} x^{c-1}\left[1+\left(\frac{x}{a}\right)^{c}\right]^{-k-1}\left\{1-\left[1+\left(\frac{x}{a}\right)^{c}\right]^{-k}\right\}^{d \alpha-1} \\
& \times\left\{1-\left\{1-\left[1+\left(\frac{x}{a}\right)^{c}\right]^{-k}\right\}^{\alpha}\right\}^{b-1}
\end{aligned}
$$


respectively, where all parameters are positive.

Further, we apply the Cramér-von Mises $\left(W^{*}\right)$ and Anderson-Darling $\left(A^{*}\right)$ statistics described in details in Chen and Balakrishnan (1995) to verify which distribution fits better to these data. Table 1 lists the MLEs, their standard errors in parentheses and the statistics $W^{*}$ and $A^{*}$. These statistics indicate that the BXIINB distribution is the best model to describe the data.

Table 1. MLEs and the measures $W^{*}$ and $A^{*}$

\begin{tabular}{|c|c|c|c|c|c|c|c|c|}
\hline \multirow{2}{*}{$\begin{array}{c}\text { Distribution } \\
\text { BXIINB }\end{array}$} & \multicolumn{5}{|c|}{ Estimates } & \multicolumn{3}{|c|}{ Statistics } \\
\hline & $\widehat{s}$ & $\widehat{\beta}$ & $\widehat{c}$ & $\widehat{k}$ & $\widehat{a}$ & & $W^{*}$ & $A^{*}$ \\
\hline & 19.1368 & 0.9607 & 2.4613 & 0.2565 & 19.7483 & & 0.0602 & 0.6047 \\
\hline & (18.9036) & $(0.0868)$ & $(0.2213)$ & (1.0065) & (23.7111) & & & \\
\hline \multirow[t]{3}{*}{ Exp-BXIIP } & $\widehat{c}$ & $\widehat{k}$ & $\widehat{a}$ & $\widehat{\alpha}$ & $\widehat{\lambda}$ & & $W^{*}$ & $A^{*}$ \\
\hline & 6.7808 & 9.5540 & 5.6803 & 0.2673 & 0.6577 & & 0.0961 & 0.6838 \\
\hline & $(0.0079)$ & (15.5828) & (1.5101) & $(0.0548)$ & (1.0049) & & & \\
\hline \multirow[t]{3}{*}{ BBXII } & $\widehat{c}$ & $\widehat{k}$ & $\widehat{a}$ & $\widehat{d}$ & $\widehat{b}$ & & $W^{*}$ & $A^{*}$ \\
\hline & 7.3601 & 5.5584 & 4.3671 & 0.2180 & 0.4961 & & 0.2317 & 1.9095 \\
\hline & $(0.0046)$ & (3.1054) & $(0.0046)$ & $(0.0278)$ & $(0.2190)$ & & & \\
\hline \multirow[t]{3}{*}{ KwBXII } & $\widehat{c}$ & $\widehat{k}$ & $\widehat{a}$ & $\widehat{d}$ & $\widehat{b}$ & & $W^{*}$ & $A^{*}$ \\
\hline & 5.9787 & 6.1332 & 6.2593 & 0.3216 & 1.8375 & & 0.0777 & 0.6050 \\
\hline & $(0.0464)$ & $(6.2411)$ & $(0.0454)$ & $(0.0529)$ & (1.1272) & & & \\
\hline \multirow[t]{3}{*}{ McBXII } & $\widehat{c}$ & $\widehat{k}$ & $\widehat{a}$ & $\widehat{d}$ & $\widehat{b}$ & $\widehat{\alpha}$ & $W^{*}$ & $A^{*}$ \\
\hline & 6.3865 & 11.0209 & 6.4909 & 0.5411 & 1.6177 & 0.5016 & 0.0939 & 0.8799 \\
\hline & $(0.0140)$ & $(0.0671)$ & $(0.0140)$ & $(0.7742)$ & $(0.3525)$ & (0.6585) & & \\
\hline
\end{tabular}

More information is provided by a visual comparison of the fitted densities to the histogram of the data. The plots of the fitted BXIINB, Exp-BXIIP, BBXII, KwBXII and McBXII density functions are displayed in Figure 4. They show that the new distribution provides a good fit to these data and that it is also a very compettitive model to the other lifetime distributions.

\section{Concluding Remarks}

We define and study a new five-parameter lifetime distribution, referred to as the Burr XII negative binomial (BXIINB) distribution, which extends several distributions widely used in the lifetime literature. The proposed model is much more flexible than the Burr XII, Weibull and log-logistic models. It is useful for modeling lifetime data with a unimodal-shaped hazard rate function. We provide a mathematical treatment of the distribution including expansions for the BXIINB density function and plots of its skewness and kurtosis. We derive explicit expressions for the ordinary and incomplete moments, generating and quantile functions, order statistics and their moments, Rényi and Shanon entropies, mean deviations and reliability. The estimation of the model parameters is approached by the method of maximum likelihood and the observed information matrix is derived. One application of the BXIINB distribution shows that it could provide a better fit than other statistical models used in lifetime data analysis.

\section{Appendix A}

We have the following result which holds for $m$ and $k$ positive integers, $\mu>-1$ and $p>0$ (Prudnikov et al., 1992, p. 21)

$$
\begin{aligned}
& I\left(p, \mu, \frac{m}{k}, v\right)=\int_{0}^{\infty} \exp (-p x) x^{\mu}\left(1+x^{\frac{m}{k}}\right)^{v} d x \\
& =\frac{k^{-v} m^{\mu+\frac{1}{2}}}{(2 \pi)^{\frac{(m-1)}{2}} \Gamma(-v) p^{\mu+1}} \times \\
& G_{k+m, k}^{k, k+m}\left(\frac{m^{m}}{p^{m}} \begin{array}{l}
\Delta(m,-\mu), \Delta(k, v+1) \\
\Delta(k, 0)
\end{array}\right),
\end{aligned}
$$

where $\Delta(k, a)=\frac{a}{k}, \frac{a+1}{k}, \cdots, \frac{a+k}{k}$. 


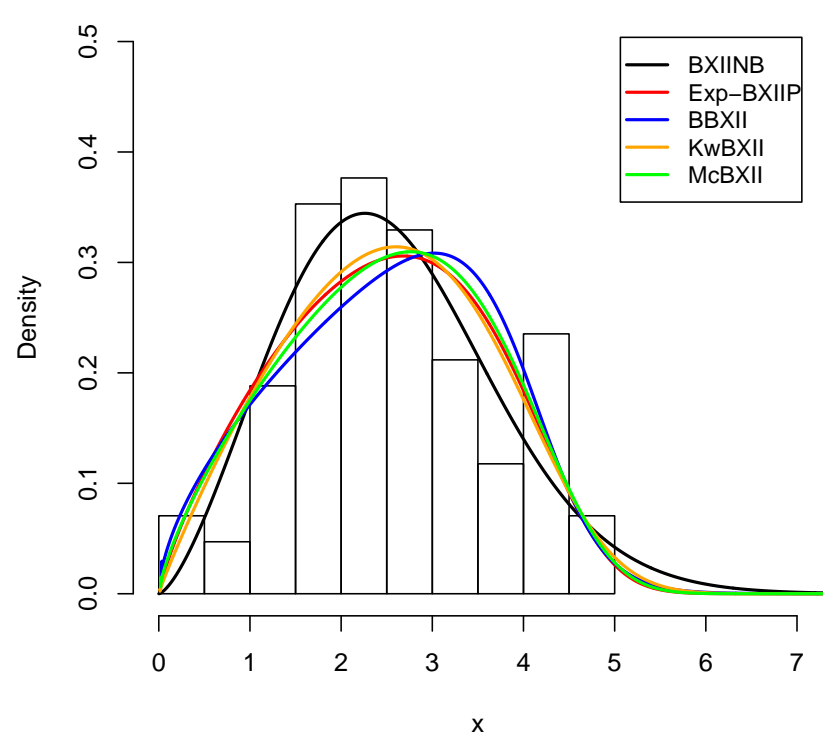

Figure 4. Some fitted densities to the histogram of the current data.

\section{Appendix B}

$$
\begin{gathered}
U_{s s}=-\frac{n\left\{\left[(1-\beta)^{s}-1\right]^{2}-s^{2}(1-\beta)^{s} \log ^{2}(1-\beta)\right\}}{s^{2}\left[(1-\beta)^{s}-1\right]^{2}}, \\
U_{s \beta}=\frac{n\left[1-(1-\beta)^{s}+s(1-\beta)^{s} \log (1-\beta)\right]}{(\beta-1)\left[(1-\beta)^{s}-1\right]^{2}}+\sum_{i=1}^{n} \frac{\left[1+\left(\frac{x_{i}}{a}\right)^{c}\right]^{-k}}{1-\beta\left[1+\left(\frac{x_{i}}{a}\right)^{c}\right]^{-k}}, \\
U_{s c}=-\sum_{i=1}^{n} \frac{\beta k\left(\frac{x_{i}}{a}\right)^{c} \log \left(\frac{x_{i}}{a}\right)\left[1+\left(\frac{x_{i}}{a}\right)^{c}\right]^{-k-1}}{1-\beta\left[1+\left(\frac{x_{i}}{a}\right)^{c}\right]^{-k}}, \\
U_{s k}=-\sum_{i=1}^{n} \frac{\beta\left[1+\left(\frac{x_{i}}{a}\right)^{c}\right]^{-k} \log \left[1+\left(\frac{x_{i}}{a}\right)^{c}\right]}{1-\beta\left[1+\left(\frac{x_{i}}{a}\right)^{c}\right]^{-k}}, \\
U_{s a}=\sum_{i=1}^{n} \frac{\beta c k x_{i}\left(\frac{x_{i}}{a}\right)^{c-1}\left[1+\left(\frac{x_{i}}{a}\right)^{c}\right]^{-k-1}}{a^{2}\left\{1-\beta\left[1+\left(\frac{x_{i}}{a}\right)^{c}\right]^{-k}\right\}}, \\
U_{\beta \beta=n}\left\{\frac{s\left[s(1-\beta)^{s}+(1-\beta)^{s}-1\right]}{\left.(\beta-1)^{2}\left[(1-\beta)^{s}-1\right]^{2}-\frac{1}{\beta^{2}}\right\}+(s+1) \sum_{i=1}^{n} \frac{\left[1+\left(\frac{x_{i}}{a}\right)^{c}\right]^{-2 k}}{\left\{1-\beta\left[1+\left(\frac{x_{i}}{a}\right)^{c}\right]^{-k}\right\}^{2}},}\right.
\end{gathered}
$$




$$
\begin{aligned}
& U_{\beta c}=-(s+1) \sum_{i=1}^{n}\left(\frac{k\left(\frac{x_{i}}{a}\right)^{c} \log \left(\frac{x_{i}}{a}\right)\left[1+\left(\frac{x_{i}}{a}\right)^{c}\right]^{-k-1}}{1-\beta\left[1+\left(\frac{x_{i}}{a}\right)^{c}\right]^{-k}}+\frac{\beta k\left(\frac{x_{i}}{a}\right)^{c} \log \left(\frac{x_{i}}{a}\right)\left[1+\left(\frac{x_{i}}{a}\right)^{c}\right]^{-2 k-1}}{\left\{1-\beta\left[1+\left(\frac{x_{i}}{a}\right)^{c}\right]^{-k}\right\}^{2}}\right) \text {, } \\
& U_{\beta k}=-(s+1) \sum_{i=1}^{n}\left(\frac{\beta\left[1+\left(\frac{x_{i}}{a}\right)^{c}\right]^{-2 k} \log \left[1+\left(\frac{x_{i}}{a}\right)^{c}\right]}{\left\{1-\beta\left[1+\left(\frac{x_{i}}{a}\right)^{c}\right]^{-k}\right\}^{2}}+\frac{\left[1+\left(\frac{x_{i}}{a}\right)^{c}\right]^{-k} \log \left[1+\left(\frac{x_{i}}{a}\right)^{c}\right]}{1-\beta\left[1+\left(\frac{x_{i}}{a}\right)^{c}\right]^{-k}}\right), \\
& U_{\beta a}=(s+1) \sum_{i=1}^{n}\left(\frac{c k x_{i}\left(\frac{x_{i}}{a}\right)^{c-1}\left[1+\left(\frac{x_{i}}{a}\right)^{c}\right]^{-k-1}}{a^{2}\left\{1-\beta\left[1+\left(\frac{x_{i}}{a}\right)^{c}\right]^{-k}\right\}}+\frac{\beta c k x_{i}\left(\frac{x_{i}}{a}\right)^{c-1}\left[1+\left(\frac{x_{i}}{a}\right)^{c}\right]^{-2 k-1}}{a^{2}\left\{1-\beta\left[1+\left(\frac{x_{i}}{a}\right)^{c}\right]^{-k}\right\}^{2}}\right), \\
& U_{c c}=\frac{n}{c^{2}}+(s+1)\left[\sum _ { i = 1 } ^ { n } \left(\frac{\beta^{2} k^{2}\left(\frac{x_{i}}{a}\right)^{2 c} \log ^{2}\left(\frac{x_{i}}{a}\right)\left[1+\left(\frac{x_{i}}{a}\right)^{c}\right]^{-2 k-2}}{\left\{1-\beta\left[1+\left(\frac{x_{i}}{a}\right)^{c}\right]^{-k}\right\}^{2}}-\frac{\beta k\left(\frac{x_{i}}{a}\right)^{c} \log ^{2}\left(\frac{x_{i}}{a}\right)}{1-\beta\left[1+\left(\frac{x_{i}}{a}\right)^{c}\right]^{-k}}\right.\right. \\
& \left.\left.\times\left[1+\left(\frac{x_{i}}{a}\right)^{c}\right]^{-k-1}-\frac{\beta k(-k-1)\left(\frac{x_{i}}{a}\right)^{2 c} \log ^{2}\left(\frac{x_{i}}{a}\right)\left[1+\left(\frac{x_{i}}{a}\right)^{c}\right]^{-k-2}}{1-\beta\left[1+\left(\frac{x_{i}}{a}\right)^{c}\right]^{-k}}\right)\right] \\
& +(k+1)\left[\sum_{i=1}^{n}\left(\frac{\left(\frac{x_{i}}{a}\right)^{c} \log ^{2}\left(\frac{x_{i}}{a}\right)}{1+\left(\frac{x_{i}}{a}\right)^{c}}-\frac{\left(\frac{x_{i}}{a}\right)^{2 c} \log ^{2}\left(\frac{x_{i}}{a}\right)}{\left[1+\left(\frac{x_{i}}{a}\right)^{c}\right]^{2}}\right)\right], \\
& U_{c k}=-\sum_{i}^{n} \frac{\left(\frac{x_{i}}{a}\right)^{c} \log \left(\frac{x_{i}}{a}\right)}{1+\left(\frac{x_{i}}{a}\right)^{c}}+(s+1) \sum_{i=1}^{n}\left(\frac{\beta^{2} k\left(\frac{x_{i}}{a}\right)^{c} \log \left(\frac{x_{i}}{a}\right) \log \left[1+\left(\frac{x_{i}}{a}\right)^{c}\right]}{\left\{1-\beta\left[1+\left(\frac{x_{i}}{a}\right)^{c}\right]^{-k}\right\}^{2}}\right. \\
& \times\left[1+\left(\frac{x_{i}}{a}\right)^{c}\right]^{-2 k-1}-\frac{\beta\left(\frac{x_{i}}{a}\right)^{c} \log \left(\frac{x_{i}}{a}\right)\left[1+\left(\frac{x_{i}}{a}\right)^{c}\right]^{-k-1}}{1-\beta\left[1+\left(\frac{x_{i}}{a}\right)^{c}\right]^{-k}}+\frac{\beta k\left(\frac{x_{i}}{a}\right)^{c} \log \left(\frac{x_{i}}{a}\right)}{1-\beta\left[1+\left(\frac{x_{i}}{a}\right)^{c}\right]^{-k}} \\
& \left.\times\left[1+\left(\frac{x_{i}}{a}\right)^{c}\right]^{-k-1} \log \left[1+\left(\frac{x_{i}}{a}\right)^{c}\right]\right), \\
& U_{c a}=-(s+1) \sum_{i=1}^{n}\left(\frac{\beta^{2} c k^{2} x_{i}\left(\frac{x_{i}}{a}\right)^{2 c-1} \log \left(\frac{x_{i}}{a}\right)\left[1+\left(\frac{x_{i}}{a}\right)^{c}\right]^{-2 k-2}}{a^{2}\left\{1-\beta\left[1+\left(\frac{x_{i}}{a}\right)^{c}\right]^{-k}\right\}^{2}}-\frac{\beta c k x_{i}\left(\frac{x_{i}}{a}\right)^{c-1}}{\left\{1-\beta\left[1+\left(\frac{x_{i}}{a}\right)^{c}\right]^{-k}\right\}}\right. \\
& \times \frac{1}{a^{2}} \log \left(\frac{x_{i}}{a}\right)\left[1+\left(\frac{x_{i}}{a}\right)^{c}\right]^{-k-1}-\frac{\beta c(-k-1) k x_{i}\left(\frac{x_{i}}{a}\right)^{2 c-1} \log \left(\frac{x_{i}}{a}\right)\left[1+\left(\frac{x_{i}}{a}\right)^{c}\right]^{-k-2}}{a^{2}\left\{1-\beta\left[1+\left(\frac{x_{i}}{a}\right)^{c}\right]^{-k}\right\}} \\
& \left.-\frac{\beta k\left(\frac{x_{i}}{a}\right)^{c}\left[1+\left(\frac{x_{i}}{a}\right)^{c}\right]^{-k-1}}{a\left\{1-\beta\left[1+\left(\frac{x_{i}}{a}\right)^{c}\right]^{-k}\right\}}\right)-(k+1) \sum_{i}^{n}\left\{\frac{c x_{i}\left(\frac{x_{i}}{a}\right)^{c-1} \log \left(\frac{x_{i}}{a}\right)}{a^{2}\left[1+\left(\frac{x_{i}}{a}\right)^{c}\right]}+\frac{\left(\frac{x_{i}}{a}\right)^{c}}{a\left[1+\left(\frac{x_{i}}{a}\right)^{c}\right]}\right. \\
& \left.-\frac{c x_{i}\left(\frac{x_{i}}{a}\right)^{2 c-1} \log \left(\frac{x_{i}}{a}\right)}{a^{2}\left[1+\left(\frac{x_{i}}{a}\right)^{c}\right]^{2}}\right\}+\frac{n}{a} \text {, }
\end{aligned}
$$




$$
\begin{aligned}
& U_{k k}=-\frac{n}{k^{2}}+(s+1) \sum_{i=1}^{n}\left(\frac{\beta^{2}\left[1+\left(\frac{x_{i}}{a}\right)^{c}\right]^{-2 k} \log ^{2}\left[1+\left(\frac{x_{i}}{a}\right)^{c}\right]}{\left\{1-\beta\left[1+\left(\frac{x_{i}}{a}\right)^{c}\right]^{-k}\right\}^{2}}+\frac{\beta\left[1+\left(\frac{x_{i}}{a}\right)^{c}\right]^{-k}}{1-\beta\left[1+\left(\frac{x_{i}}{a}\right)^{c}\right]^{-k}}\right. \\
& \left.\times \log ^{2}\left[1+\left(\frac{x_{i}}{a}\right)^{c}\right]\right), \\
& U_{k a}=\sum_{i=1}^{n} \frac{c x_{i}\left(\frac{x_{i}}{a}\right)^{c-1}}{a^{2}\left[1+\left(\frac{x_{i}}{a}\right)^{c}\right]}-(s+1) \sum_{i=1}^{n}\left(\frac{\beta^{2} c k x_{i}\left(\frac{x_{i}}{a}\right)^{c-1}\left[1+\left(\frac{x_{i}}{a}\right)^{c}\right]^{-2 k-1} \log \left[1+\left(\frac{x_{i}}{a}\right)^{c}\right]}{a^{2}\left\{1-\beta\left[1+\left(\frac{x_{i}}{a}\right)^{c}\right]^{-k}\right\}^{2}}\right. \\
& \left.-\frac{\beta c x_{i}\left(\frac{x_{i}}{a}\right)^{c-1}\left[1+\left(\frac{x_{i}}{a}\right)^{c}\right]^{-k-1}}{a^{2}\left\{1-\beta\left[1+\left(\frac{x_{i}}{a}\right)^{c}\right]^{-k}\right\}}+\frac{\beta c k x_{i}\left(\frac{x_{i}}{a}\right)^{c-1}\left[1+\left(\frac{x_{i}}{a}\right)^{c}\right]^{-k-1} \log \left[1+\left(\frac{x_{i}}{a}\right)^{c}\right]}{a^{2}\left\{1-\beta\left[1+\left(\frac{x_{i}}{a}\right)^{c}\right]^{-k}\right\}}\right), \\
& U_{a a}=\frac{c n}{a^{2}}-(s+1) \sum_{i=1}^{n}\left(-\frac{\beta^{2} c^{2} k^{2} x_{i}^{2}\left(\frac{x_{i}}{a}\right)^{2 c-2}\left[1+\left(\frac{x_{i}}{a}\right)^{c}\right]^{-2 k-2}}{a^{4}\left\{1-\beta\left[1+\left(\frac{x_{i}}{a}\right)^{c}\right]^{-k}\right\}^{2}}+\frac{\beta c^{2}(-k-1) k}{\left\{1-\beta\left[1+\left(\frac{x_{i}}{a}\right)^{c}\right]^{-k}\right\}}\right. \\
& \times \frac{x_{i}^{2}}{a^{4}}\left(\frac{x_{i}}{a}\right)^{2 c-2}\left[1+\left(\frac{x_{i}}{a}\right)^{c}\right]^{-k-2}+\frac{\beta c k(c-1) x_{i}^{2}\left(\frac{x_{i}}{a}\right)^{c-2}\left[1+\left(\frac{x_{i}}{a}\right)^{c}\right]^{-k-1}}{a^{4}\left\{1-\beta\left[1+\left(\frac{x_{i}}{a}\right)^{c}\right]^{-k}\right\}} \\
& \left.+\frac{2 \beta c k x_{i}\left(\frac{x_{i}}{a}\right)^{c-1}\left[1+\left(\frac{x_{i}}{a}\right)^{c}\right]^{-k-1}}{a^{3}\left\{1-\beta\left[1+\left(\frac{x_{i}}{a}\right)^{c}\right]^{-k}\right\}}\right)+(k+1) \sum_{i=1}^{n}\left(\frac{c^{2} x_{i}^{2}\left(\frac{x_{i}}{a}\right)^{2 c-2}}{a^{4}\left[1+\left(\frac{x_{i}}{a}\right)^{c}\right]^{2}}-\frac{c(c-1)}{\left[1+\left(\frac{x_{i}}{a}\right)^{c}\right]}\right. \\
& \left.\times \frac{x_{i}^{2}}{a^{4}}\left(\frac{x_{i}}{a}\right)^{c-2}-\frac{2 c x_{i}\left(\frac{x_{i}}{a}\right)^{c-1}}{a^{3}\left[1+\left(\frac{x_{i}}{a}\right)^{c}\right]}\right) \text {. }
\end{aligned}
$$

\section{References}

Chen, G., \& Balakrishnan, N. (1995). A general purpose approximate goodness-of-fit test. Journal of Quality Technology, 27, 154-161.

Doornik, J. A. (2006). An Object-Oriented Matrix Language-Ox 4, 5th ed. Timberlake Consultants Press: London.

Gradshteyn, I. S., \& Ryzhik, I. M. (2000). Table of Integrals, Series and Products. Academic Press, San Diego.

Kenney, J. F., \& Keeping, E. S. (1962). Mathematics of Statistics, Part 1, 3rd ed. Princeton, New Jersey.

Moors, J. J. (1988). A quantile alternative for kurtosis. Journal of the Royal Statistical Society D, 37, 25-32.

Murthy, D. N. P., Xie, M., \& Jiang, R. (2004). Weibull Models, John Wiley and Sons, New Jersey.

Parnaiba, P. F. P., Ortega, E. M. M., Cordeiro, G. M., \& Pescim, R. R. (2011). The beta Burr XII distribution with application to lifetime data. Computational Statistics and Data Analysis, 55, 1118-1136. http://dx.doi.org/10.1016/j.csda.2010.09.009

Parnaiba, P. F. P., Ortega, E. M. M., Cordeiro, G. M., \& Pascoa, M. A. R. (2012). The Kumaraswamy Burr XII distribution: Theory and Practice. Journal of Statistical Computation and Simulation, 82, 1-27.

Percontini, A., Cordeiro, G. M., \& Bourguignon, M. (2013). The G-Negative Binomial Family: General Properties and Applications. Advances and Applications in Statistics, 35, 127-160.

Prudnikov, A. P., Brychkov, Y. A., \& Marichev, O. I. (1986). Integrals and Series, volume 1. Gordon and Breach Science Publishers, Amsterdam. 
Prudnikov, A. P., Brychkov, Y. A., \& Marichev, O. I. (1992). Integrals and Series, volume 4. Gordon and Breach Science Publishers, Amsterdam.

Rényi, A. (1961). On measures of entropy and information. In: Proceedings of the 4th Berkeley Symposium on Mathematical Statistics and Probability, I, 547-561. University of California Press: Berkeley.

Shannon, C. E. (1951). Prediction and entropy of printed English. The Bell System Technical Journal, 30, 50-64. http://dx.doi.org/10.1002/j.1538-7305.1951.tb01366.x

Shao, Q. (2004a). Notes on maximum likelihood estimation for the three-parameter Burr XII distribution. Computational Statistics and Data Analysis, 45, 675-687. http://dx.doi.org/10.1016/S0167-9473(02)00367-5

Shao, Q., Wong, H., \& Xia, J. (2004b). Models for extremes using the extended three parameter Burr XII system with application to flood frequency analysis. Hydrological Sciences (Journal des Sciences Hydrologiques), 49, 685-702. http://dx.doi.org/10.1623/hysj.49.4.685.54425

Silva, G. O., Ortega, E. M. M., Garibay, V. C., \& Barreto, M. L. (2008). Log-Burr XII regression models with censored Data. Computational Statistics and Data Analysis, 52, 3820-3842. http://dx.doi.org/10.1016/j.csda.2008.01.003

Silva, G. O., Ortega, E. M. M., \& Paula, G. A. (2011). Residuals for log-Burr XII regression models in survival analysis. Journal of Applied Statistics, 38, 1435-1445. http://dx.doi.org/10.1080/02664763.2010.505950

Soliman, A. A. (2005). Estimation of Parameters of Life from Progressively Censored Data using Burr-XII Model. IEEE Transactions on Reliability, 54, 34-42. http://dx.doi.org/10.1109/TR.2004.842528

Wu, S. J., Chen, Y. J., \& Chang, C. T. (2007). Statistical inference based on progressively censored samples with random removals from the Burr type XII distribution. Journal of Statistical Computation and Simulation, 77, 19-27. http://dx.doi.org/10.1080/10629360600569204

Zimmer, W. J., Keats, J. B., \& Wang, F. K. (1998). The Burr XII distribution in reliability analysis. Journal of Quality Technology, 30, 386-94.

\section{Copyrights}

Copyright for this article is retained by the author(s), with first publication rights granted to the journal.

This is an open-access article distributed under the terms and conditions of the Creative Commons Attribution license (http://creativecommons.org/licenses/by/3.0/). 